\title{
Sexually transmitted diseases in a defined population of women
}

\author{
M W ADLER, E M BELSEY, J S ROGERS
}

\begin{abstract}
A study was conducted to identify and estimate the proportion of patients sufiering from gonorrhoea, trichomoniasis, and candidosis, both with and without symptoms, seeking care or failing to seek care at all. Samples of women in a defined population were studied in antenatal, gynaecology, family planning, and sexually transmitted diseases clinics and in general practice. The incidence rates varied according to the conditions and to whether cases not proved microbiologically were included or excluded. The incidence rate may be less important than the prevalence rate since the former takes into account patients who have sought care whereas the latter is largely contributed by asymptomatic women who do not consult. The highest prevalence rates, in different agencies, were found for candidosis followed by trichomoniasis, with very low or zero rates for gonorrhoea.

In view of these results general practitioners could treat women with genital symptoms empirically so long as accurate sexual histories are taken and follow-up were guaranteed. There is no place for wide-scale screening for gonorrhoea, but limited screening for trichomoniasis in antenatal, gynaecology, and hospital family planning clinics should be encouraged.
\end{abstract}

Academic Department of Genitourinary Medicine, Middlesex Hospital Medical School, London W1N 8AA

$M$ W ADLER, MD, MRCP, professor

E M BELSEY, MSC, BSC, research statistician and honorary lecturer J S ROGERS, SRN, research nurse

\section{Introduction}

The routine published statistics for the sexually transmitted diseases are derived from the returns of cases treated made from clinics for sexually transmitted diseases in the United Kingdom each year. These are an underestimate of cases since they omit three categories of patients-those treated in general or private practice, or in antenatal and gynaecology clinics; symptomless patients who may never be treated; and patients with symptoms who decide to ignore them.

In the past, surveys carried out in the United Kingdom to calculate the amount of gonorrhoea, trichomoniasis, and candidosis found in gynaecology, antenatal, and family planning clinics ${ }^{1-8}$ were limited to specific age groups and to patients who had opted to seek medical care, often for an unrelated condition. Since they refer only to one part of the total population of women at risk of contracting a sexually transmitted disease, these findings cannot be related to a defined population at risk, and correct incidence or prevalence rates cannot be obtained. In view of the potential shortfall in the current statistics, as outlined, and the ad-hoc nature of past surveys we studied the incidence and prevalence of gonorrhoea, trichomoniasis, and candidosis in a defined population of women.

\section{Methods}

\section{PATIENTS AND SAMPLING}

The study was designed to identify patients both with and without symptoms seeking care or failing to seek care at all. It was limited to women since in them sexually transmitted diseases are more often asymptomatic, and thus potentially untreated. Additionally, we envisaged considerable difficulty in obtaining a sample of asymptomatic men.

The different pathways that may be taken by a woman with sexually transmitted disease and the agencies sampled in the current study are 
shown in the figure. It was expected that patients with genital symptoms could seek care from general practitioners, sexually transmitted diseases clinics, or be referred to gynaecology clinics. In contrast, symptomless women, who are unaware of disease, will not consult but could be identified by screening carried out in antenatal and family planning clinics and general practice. The divisions between symptomatic and symptomless patients and the ways in which they may be identified are arbitary. For example, patients who ignore their

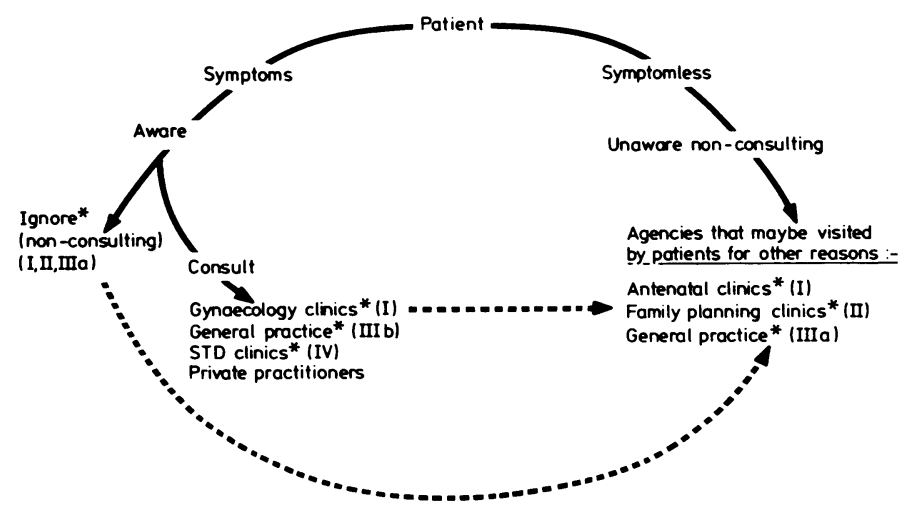

Pathway of patient with a sexually transmitted disease and agencies sampled (*) in study.

symptoms could be picked up by screening in the three agencies just mentioned, while not all women attending gynaecology clinics do so with symptoms due to a sexually transmitted disease and could fall into the category of non-consulting patients. Overlap does occur, and this theoretical division has been created only as a means of identifying the different agencies that need to be sampled if a total picture of these diseases is to be obtained.

The study area was defined as the health area of Brent and Harrow and the population as those people residing within this area. The 1979 projected female population was 209100 , of whom 116100 were aged 16-59.9 This area, in North-west London, comprises a "wedge" of suburban London, and the reasons for choosing it were its broad ethnic and social mix and the extensive contacts with hospital and community staff developed from research.

It was not considered necessary to examine all the women and agencies in the study area. Instead, samples of patients aged from 16 to 59 inclusive were screened and included in the study when they attended the following.

I Antenatal and gynaecology clinics-Three hospitals, of varying size, provide antenatal and gynaecology services. All patients attending each clinic for the first time over a three-month period were asked to take part.

II Family planning clinics-Three family planning clinics were chosen to be representative of the different geographical and demographic characteristics known to exist in Brent and Harrow. A sample of 300 consecutive patients, equally split among the three clinics, was examined.

III General practice-The general practice aspect of the study was divided into two parts: (a) screening of women not consulting whom it was thought would be predominantly asymptomatic but who might have symptoms that they chose to ignore; and $(b)$ recording and counting of women consulting with genital symptoms.

The screening of non-consulting women was carried out in a practice of four doctors in Perivale. The practice age-sex register was used to select a one in two sample, to whom a letter was sent offering an "additional service for health problems that can affect women." It was pointed out that those who were "healthy" should come as well as those who had symptoms. Cervical cytology and counselling were offered. Up to three letters were sent to each patient, and if there was still no response as many as three household visits were made.

The second part of the general practice study, concerned with the quantification of patients consulting with genital symptoms, was carried out in nine practices containing 15 general practitioners. The sample of practices was stratified by geographical area, size of practice, and whether or not the doctors provided family planning services.

\section{DATA COLLECTION}

A standard method of collecting data and examining patients was used in antenatal, gynaecology, and family planning clinics (I and II) and for screening non-consulting women in general practice (IIIa). All the women were asked to complete a self-administered questionnaire that recorded demographic data, information about present and past genital symptoms and their treatment, if any, and current contraceptive use. Two research nurses were available to explain the study, check the questionnaire with patients, and then carry out the appropriate tests and observations, which were recorded on a standard form. A Cusco's bivalve speculum was passed on each patient and specimens from the posterior fornix and lateral vaginal wall were innoculated in Feinberg-Whittington medium in an attempt to isolate Trichomonas vaginalis and Candida albicans. Specimens from the cervix and urethra were inoculated on to columbia blood agar, an appropriate media for culturing Neisseria gonorrhoeae. Wet preparations and Gram-staining were not performed. Microbiology was performed in our own laboratory.

Since so many general practitioners in various parts of the study area were concerned in the quantification of women consulting with genital symptoms (IIIb) it was not feasible for the research nurses to examine the patients as described above. Practitioners recorded patient's symptoms, the results of examinations and bacteriological tests, if carried out, and treatment. This was done for two two-week periods at different times of the year. Thus this aspect of the survey was the "softest" part of our work.

IV Disease treated in sexually transmitted disease clinics-A proportion of women residing in the study area will have obtained treatment for their disease from such a clinic, either within or outside the study area. Information about cases treated within the study area was obtained from the one existing clinic based at Central Middlesex Hospital. Information about women resident in the study area who had sought care in any other clinic outside the area, but in London, was also obtained. These data were derived from a study of a 1 in 40 sample of patients at sexually transmitted diseases clinics in England and Wales for 1978, which was designed to estimate the patient/case ratio for all sexually transmitted diseases. ${ }^{10}$

\section{Results}

\section{RESPONDENTS}

Altogether 1838 consecutive patients were approached in the antenatal, gynaecology, and family planning clinics, of whom 80 $(4.3 \%)$ refused to be examined and have tests taken by the nurses. As might be expected, the response rate was lower (65\%) among nonconsulting women invited to the surgery for screening during the general practice phase of the study. The response rate within general practice is examined in greater detail elsewhere ( $M \mathbb{W}$ Adler, unpublished observation).

\section{INCIDENCE AND PREVALENCE}

The study was designed to sample both patients who sought care for genital symptoms and those who had not either because they ignored their symptoms or were asymptomatic. The group of women seeking care indicates the incidence of diseases (number of new cases that occur during a specified period) and those who have not sought care but are identified as a result of screening indicate the point prevalence (number of cases in the community at a specified point in time).

\section{Incidence}

Table I shows the calculated incidence over a 12-month period. The 15 general practitioners, with a total list size of 34258 , saw 3710 women, aged $16-59$, during the two sampling periods. Of these women, $97(2.6 \%)$ presented with genital symptoms, and four cases of candidosis and three of trichomoniasis were established by microbiological tests. No cases of gonorrhoea were discovered. Some of the 97 patients with symptoms were not investigated but nevertheless were treated on empirical grounds. The diagnoses in such patients were based on the treatment prescribed by the practitioners (33 candidosis, 2 trichomoniasis, 1 gonorrhoea). On the basis of these findings it was calculated that in one year the 239 practitioners 


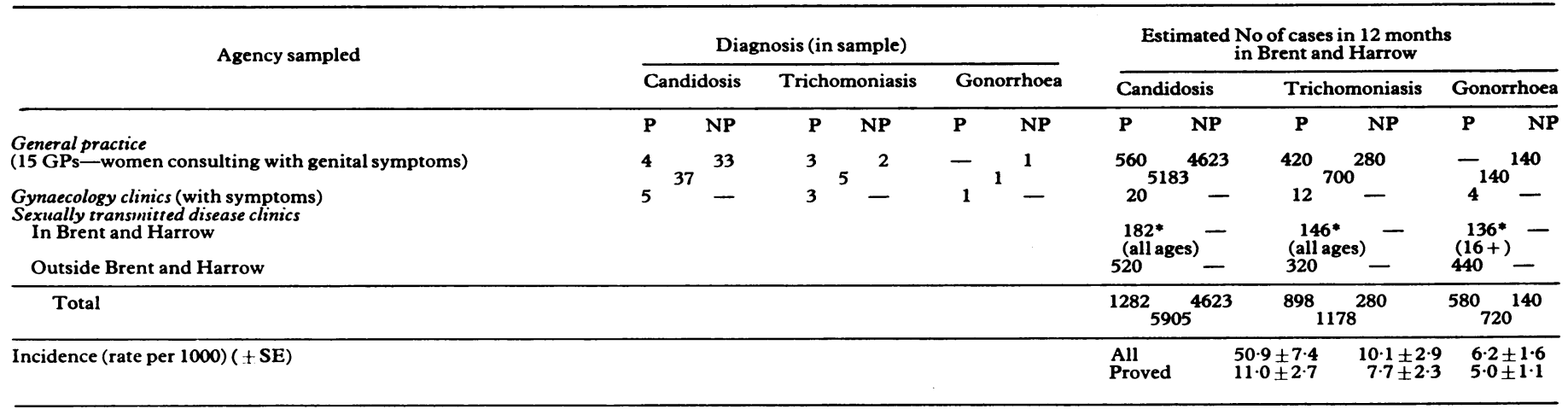

*Actual number 1978 (official clinic returns to DHSS).

$\mathbf{P}=$ Proved.

NP $=$ Not proved.

TABLE II-Prevalence of candidosis, trichomoniasis, and gonorrhoea in Brent and Harrow

\begin{tabular}{|c|c|c|c|c|c|c|c|}
\hline \multirow{2}{*}{ Agency sampled } & \multirow{2}{*}{$\begin{array}{c}\text { No } \\
\text { of patients } \\
\text { seen }\end{array}$} & \multicolumn{3}{|c|}{ Diagnosis in sample } & \multicolumn{3}{|c|}{ Estimated prevalence rates $( \pm S E) / 1000$} \\
\hline & & Candidosis & Trichomoniasis & Gonorrhoea & Candidosis* & Trichomoniasis $\dagger$ & Gonorrhoea \\
\hline $\begin{array}{l}\text { General practice } \\
\text { Women not consulting-invited to attend } \\
\text { Antenatal } \\
\text { Gynaecology (no symptoms) } \\
\text { Family planning }\end{array}$ & $\begin{array}{l}528 \\
903 \\
480 \\
300\end{array}$ & $\begin{array}{r}51 \\
114 \\
43 \\
15\end{array}$ & $\begin{array}{l}15 \\
33 \\
32 \\
13\end{array}$ & $\begin{array}{l}0 \\
3 \\
2 \\
0\end{array}$ & $\begin{array}{l}93 \cdot 2 \pm 12 \cdot 9 \\
83 \cdot 1 \pm 10 \cdot 5 \\
78 \cdot 0 \pm 13 \cdot 2 \\
36 \cdot 4 \pm 9 \cdot 3\end{array}$ & $\begin{array}{l}32 \cdot 4 \pm 8 \cdot 6 \\
44 \cdot 4 \pm 8 \cdot 6 \\
69 \cdot 6 \pm 11 \cdot 9 \\
46 \cdot 9 \pm 12 \cdot 5\end{array}$ & $\begin{array}{c}0.0 \\
3 \cdot 3 \pm 1.9 \\
4 \cdot 2 \pm 2 \cdot 9 \\
0.0\end{array}$ \\
\hline
\end{tabular}

*Standardised by age distribution of female population Brent and Harrow, 1979.
+Standardised by marital state distribution of female population Brent and Harrow, 1971.

working in the Brent and Harrow area, with a total female population aged 16-59 of 116100 , would treat 5183 cases of candidosis, of which only 560 would be proved microbiologically. Likewise, 700 cases of trichomoniasis would be treated but only 420 would be proved; the figures for gonorrhoea are 140 cases treated, none of which would be established microbiologically.

In a three-month period in the gynaecology clinics 555 patients were screened. Of these, 75 had been referred with symptoms possibly due to a genital infection and were therefore included in the calculation of incidence; five were found to be suffering from candidosis, three from trichomoniasis, and one from gonorrhoea. If the consultation and disease rates were constant throughout the year, 20 cases of candidosis, 12 of trichomoniasis, and four of gonorrhoea would be identified in 12 months.

The final group of patients who contribute towards the incidence are those resident in the Brent and Harrow area seen in sexually transmitted diseases clinics both within and outside the area. During the whole of 1978,182 cases of candidosis, 146 of trichomoniasis, and 136 of gonorrhoea were diagnosed among patients attending the one sexually transmitted diseases clinic within the study area. During the same year, the estimated numbers of cases arising from women resident in Brent and Harrow but seeking care in other London clinics were 520 of candidosis, $\mathbf{4 4 0}$ of gonorrhoea, and 320 of trichomoniasis.

The incidence rates per 1000 women aged 16-59 in Brent and Harrow vary according to whether cases not proved microbiologically are included or excluded. The incidence rate for all cases of candidosis is calculated to be 50.9 per 1000 , for trichomoniasis 10.1 per 1000 , and for gonorrhoea 6.2 per 1000 . If the calculation is limited only to microbiologically proved cases the rates naturally decrease. Thus for candida the incidence rate is 11.0 per 1000 , for trichomoniasis 7.7 per 1000 , and for gonorrhoea $5 \cdot 0$ per 1000 .

\section{Prevalence}

The prevalence of disease may be calculated from the cases found in women who were not seeking care for genital symptoms when screened. All the cases included in the estimates of prevalence were proved microbiologically. In contrast to the incidence rates, however, which were calculated by summing cases diagnosed in women consulting at various agencies, the prevalence rates could have been biased since they are based solely on cases identified at screening, and rest on the assumption that the women who participated were representative of the female population of Brent and Harrow. Comparisons between the distributions of age and marital state among patients sampled in the different agencies and those in the female population of Brent and Harrow confirmed that they were significantly different. The prevalence rates for candidosis were therefore standardised by age, since this condition was found to be related to age (patients with the condition were younger than those without), while the rates for trichomoniasis were standardised by marital state (more single patients suffered from the condition). No standardisation was performed for gonorrhoea since there were too few cases. Calculation of overall population prevalence rates for the three diseases was not thought to be justified, since the weights that would have to be assigned to each component rate are unknown.

Table II shows numbers of women screened in general practice and antenatal, gynaecology, and family planning clinics and the numbers of positive diagnoses. Of the 555 women examined in gynaecology clinics, $\mathbf{4 8 0}$ were referred for symptoms unrelated to sexually transmitted disease and are therefore included in the calculation of prevalence. None of the women screened in antenatal and family planning clinics were attending because of genital symptoms. The prevalence of the three conditions varies among agencies: for candidosis the highest rates were found in general practice and antenatal clinics; for trichomoniasis the highest rate was in gynaecological patients with lower but similar rates in antenatal and family planning patients. No cases of gonorrhoea were identified in general practice or family planning clinics. The prevalence rates among antenatal and gynaecological patients were similar, although the latter was the higher.

\section{Discussion}

The incidence and prevalence of three diseases have been reported. In our study both rates were highest for candidosis, particularly among general practice patients. In view of the poor discriminating power of genital symptoms women in whom candidosis is diagnosed and treated empirically may, in fact, be suffering from trichomoniasis or gonorrhoea, or both. ${ }^{11} 12$ Given that candidosis is considerably more common, how should general practitioners manage women presenting with genital symptoms? One approach is to advise practitioners that 
all such women be referred to a sexually transmitted diseases clinic for full microbiological tests or that these are done by the practitioner in the surgery. The strategy of referring all patients with genital symptoms to clinics may be desirable, but it is not practicable. It would result in extensive increases in clinic work load, the diagnosis of candidosis, and the attendance of low-risk women. A more pragmatic strategy would be for general practitioners to take accurate sexual histories in an attempt to identify high-risk individuals (recent partner change, multiple sexual contacts, recurrent or persistent symptoms, and symptoms in sexual partners). These patients could then be referred for specialist advice. The remaining patients could be treated empirically for candidosis. This strategy, however, should be followed only if the patient is advised to return, so that persistent symptoms may be fully investigated in the practice or a clinic to exclude trichomoniasis and gonorrhoea. Additionally, since gonorrhoea is always and trichomoniasis usually spread by sexual intercourse, male contacts need to be traced, investigated, and treated.

The other question to be answered is whether the findings of this study indicate the existence of such a substantial amount of undetected and untreated disease that alterations in strategy for controlling sexually transmitted diseases are required. The incidence rates calculated in this study take into account patients who have sought treatment for a potential sexually transmitted disease. It is therefore more important to consider the prevalence rates, which are largely contributed by asymptomatic women who, since they do not consult, remain outside the medical care system. The rates and clinical importance, however, of the three diseases differ considerably. The prevalence of gonorrhoea was either zero or under five per 1000 in the different agencies sampled. Although this is the most serious of the three diseases in terms of complications and infectivity, such low rates hardly justify wide-scale screening for this condition among women attending general practice, antenatal, gynaecology, and family planning clinics.

The prevalence rates for trichomoniasis were higher than for gonorrhoea and varied among clinics: gynaecology (70 per 1000), family planning (47), antenatal (44), and general practice (32). Consideration needs to be given to whether this is sufficiently common to recommend screening all women for trichomoniasis. In numerical terms these rates appear high, but the decision to screen depends largely on the consequences of non-treatment and the ease with which screening can be carried out. Even though the symptoms of trichomonal infection can be severe, the disease is not serious since salpingitis, metastatic complications, and fetal infection do not occur. Establishing the diagnosis by the dark ground examination of a wet preparation of material taken from the posterior fornix is relatively easy. Given the frequency with which this disease was found among women sampled in hospital, particularly in gynaecology clinics, and the ease of immediate microscopic diagnosis, it is probably justifiable to look for this condition in all women attending antenatal, gynaecology, and hospital family planning clinics.

The prevalence rates for candidosis were the highest of the three diseases. Many women with this condition are asymptomatic. Since there is still controversy about the appropriateness of treating asymptomatic candidosis, and the disease has no serious complications, screening is not recommended. ${ }^{13}$

The sample of women studied was unselected in that it was drawn from several different agencies, and from those who had and had not sought care. Those studied, however, are not necessarily representative of women of similar ages in the rest of the United Kingdom or Brent and Harrow. Studies in other parts of the country would be required to estimate national incidence and prevalence rates. The problem caused by differences found in terms of age and marital state between the study patients and the Brent and Harrow population from which they were drawn was partially solved by the standardisation carried out. Nevertheless, this does not overcome the bias potentially caused by the low response rate in one part of the study (general practice screening). Experience from other screening surveys, such as for cervical cytology, suggest that disease or abnormalities are higher in non-respondents. ${ }^{15}$ Thus our rates may be slightly low.

The findings of this study would suggest that wide-scale $\stackrel{\varnothing}{\odot}$ screening for gonorrhoea is not justified but that limited screen- $c$ ing for trichomoniasis within the context of antenatal, gynae- $\frac{\Omega}{\overline{3}}$ cology, and hospital family planning clinics should be en- $\bar{J}$ couraged. The decision not to screen for gonorrhoea should be taken only if two criteria are fulfilled. Firstly, good comprehensive facilities for contact tracing must exist so that asympto- 0 matic women not seeking care will be examined, and, secondly, general practitioners engaging in empirical treatment without $\stackrel{\overline{\vec{S}}}{\rightarrow}$ microbiological support should follow up all their patients thus treated to ensure that trichomoniasis and gonorrhoea are not present, and that subsequent microbiological tests and contact $\frac{\bar{F}}{\frac{F}{\sigma}}$ tracing are performed.

This study was supported as part of a research contract from the $\vec{\theta}$ Medical Research Council. We thank all the clinicians and patients? who took part. We are grateful to Professors D L Miller and G Rose $\vec{\omega}$ for their comments.

\section{References}

${ }^{1}$ Driscoll AM, McCoy DR, Nicol CS, Barrow J. Sexually transmitted N disease in gynaecological outpatients with vaginal discharge. Br $\mathcal{F}$ Vener

Dis $1970 ; 46: 125$.
2 Thin RNT, Michael AN. Sexually transmitted disease in antenatal patients. BrF Vener Dis $1970 ; 46 ; 126-8$.

3 Hughes WH, Davies JM. Better specimens from the female genital tract.

Br Med F 1971 ;iv:424-5.
4 Rees DA, Hamlett JD. Screening for gonorrhoea in pregnancy. Fournal $\cong$ of Obstrics and Gynaecology of the British Commonwealth 1972;79:344-7.

5 Cassie R, Stevenson A. Screening for gonorrhoea, trichomoniasis moni- $\vec{c}$ liasis and syphilis in pregnancy. Fournal of Obstetrics and Gynaecology $\underset{\rightarrow}{\infty}$ of the British Commonwealth $1973 ; 80: 48-51$.

- Silverstone PI, Snodgrass CA, Wigfield AS. Value of screening for gonorrhoea in obstetrics and gynaecology. $\mathrm{Br} \mathcal{F}$ Vener Dis 1974;50: 53-6.

7 Sparks RA, Williams GL, Boyce JMH, Fitzgerald TC, Shelley G. $\overline{\mathrm{O}}$ Antenatal screening for candidiasis, trichomoniasis, and gonorrhoea. 은 Br ₹ Vener Dis $1975 ; 61: 110-5$.

${ }^{8}$ Nabarro JM, Grant AM, Simon R, Beral V, Catterall RD. Screening for $\stackrel{\varrho}{\vec{t}}$ gonorrhoea in a central London family planning clinic. Fertility and $\mathrm{O}$ Contraception 1978;2:1-4.

9 Population estimates for 1979. London: North-west Thames Regional Health Authority, 1980. (Paper No 6.)

10 Belsey EM, Adler MW. A study of STD clinic attenders in England and Wales, 1978: 1 Patients versus cases. Brf Vener Dis (in press).

11 Wright HS, Palmer A. The prevalence and clinical diagnosis of vaginal candidosis in non-pregnant patients with vaginal discharge and pruritis vulvae. $7 R$ Coll Gen Pract 1978;28:719-23.

12 Oriel JD, Partridge BM, Denny MS, Coleman JC. Genital yeast infections. Br MedF 1972;iv:761-4.

${ }^{13} \mathrm{King} \mathrm{A,} \mathrm{Nicol} \mathrm{C,} \mathrm{Rodin} \mathrm{P.} \mathrm{Venereal} \mathrm{diseases.} \mathrm{4th} \mathrm{ed.} \mathrm{London:} \mathrm{Bailliére} \frac{}{3}$ Tindall, 1980.

14 Adelstein AM, Husain OAN, Spriggs AI. Cancer of cervix and screening. $\frac{\mathrm{J}}{\mathrm{J}}$ Br Med F 1981;282:564.

(Accepted 20 May 1981)

Is there a test that could help to decide whether or not a woman approach- $\stackrel{\oplus}{\stackrel{\Phi}{+}}$ ing the menopause could stop taking the pill?

I do not know of such a test. Follicle-stimulating hormone blood $\stackrel{\mathbb{D}}{\mathbb{Q}}$ concentration is high in the menopause and is probably the best $\overrightarrow{\mathbb{D}}$ indicator of the end of ovulation. If, however, the patient has reached $\frac{a}{\sigma}$ the stage where she can no longer ovulate but is still taking the contraceptive pill the follicle-stimulating hormone concentration may $\delta$ not be abnormally high, the oestrogen in the pill reducing its secretion. This patient should stop taking the pill and use a barrier method of contraception. If after a few months she has had no periods, has menopausal symptoms, and the follicle-stimulating hormone concentration is high it would be perfectly safe to stop all contraception. 